\title{
Analysis of Energy Dissipation Characteristics of Damaged Sandstone under Impact Load
}

\author{
Feng Wang $\mathbb{D},{ }^{1,2,3}$ Haibo Wang $\mathbb{D},,^{1,2}$ Ying Xu $\mathbb{D}^{1,2}$ Bing Cheng $\mathbb{D},{ }^{1,2}$ and Qianqian Wang ${ }^{1,2}$ \\ ${ }^{1}$ State Key Laboratory of Mining Response and Disaster Prevention and Control in Deep Coal Mines, \\ Anhui University of Science and Technology, Huainan, Anhui 232001, China \\ ${ }^{2}$ School of Civil Engineering and Architecture, Anhui University of Science and Technology, Huainan, Anhui 232001, China \\ ${ }^{3}$ Beijing CAS-Mechanics Blasting Co., Ltd., Beijing 101318, China
}

Correspondence should be addressed to Haibo Wang; wanghb_aust@163.com

Received 24 June 2021; Accepted 15 July 2021; Published 22 July 2021

Academic Editor: Xiao Wang

Copyright (C) 2021 Feng Wang et al. This is an open access article distributed under the Creative Commons Attribution License, which permits unrestricted use, distribution, and reproduction in any medium, provided the original work is properly cited.

\begin{abstract}
Before rock burst, coal, and gas outburst dynamic load, rock mass in geotechnical engineering has been an indifferent degree of damage. The dissipation energy of rock mass under dynamic load reflects the difficulty of rock breaking. In view of the energy dissipation of damaged rock mass under dynamic load, the cyclic loading and unloading test is carried out to make sandstone in different damage states, and the damage degree of sandstone is characterized by the change of longitudinal wave velocity before and after cyclic loading and unloading. Then, the rock with different damage degrees is tested by adopting the split Hopkinson pressure bar (SHPB). Finally, the energy dissipation characteristics of damaged rock under impact load are analyzed. The results show that the damage factor of sandstone increases with the increase of the upper limit of stress after cyclic static loading. The dynamic strength and peak strain of damaged sandstone increase with the increase of impact pressure and decrease with the increase of damage degree. With the increase of damage degree of sandstone, the reflection energy and dissipation energy of sandstone increase, while the transmission energy decreases.
\end{abstract}

\section{Introduction}

As a common bearing medium in nature, rock mass widely exists in geotechnical engineering such as tunnel, subway, rock slope, and coal mine roadway [1-7]. In the long period of geological history, the rock has experienced the geological tectonic movement, so that there are different degrees of joints, fissures, and other weak surfaces in the rock, and in different degrees of damage state [8-12]. In modern times, affected by the disturbance of rock burst [13], coal and gas outburst [14], and human excavation activities [15], the damaged rock mass began to destroy and lose stability under the action of dynamic load, which caused great hidden danger to the safety of engineering production and human life [16]. Due to the different stress history of the bearing rock mass, the damage caused by the weak surface is different, and the dynamic response of the rock with different damage degrees is also different [17]. Therefore, it is necessary to analyze the dynamic mechanical properties of damaged rock under impact load.

At present, the analysis of mechanical properties of damaged rock mainly focuses on the static mechanical properties, including the strength [18], deformation [19], and damage degree of damaged rock [20]. As for the dynamic response of damaged rock under impact load, there are not many types of research at present, and there is still room for further research. Different from the static mechanical properties, the dynamic mechanical properties and dynamic response of brittle materials such as rock under impact load are more sensitive to the change of strain rate $[21,22]$. With the action of static load, the medium inside the rock is in a state of relative balance, and the inertial effect caused by the vibration between the medium can be ignored [23]. However, the duration of impact load acting on rock is very short, so the inertial interaction between media in this time scale cannot be ignored. Based on the dynamic 
constitutive relationship, dynamic strength change, strain rate sensitivity, and elastic modulus of rock, many scholars carry out dynamic mechanical tests [24-27].

At present, the research on dynamic mechanical properties of rock is mainly carried out by means of an SHPB experimental device [28]. In the general study of rock dynamics, the analysis of strain rate sensitivity of rock under impact load is the focus of the study. By analyzing the mechanical characteristics of damaged rock under different strain rates, the mechanical behavior of rock under different dynamic loads in geotechnical engineering is studied [29]. In the 1960s, Kumar carried out impact tests on granite and basalt with the SHPB and found the strain rate effect of rock under impact load for the first time [30]. After that, many scholars have carried out impact dynamic tests on rocks of other lithology and found that rock-like brittle materials are highly sensitive to strain rate when subjected to impact load [31]. For the rock with weak planes structure such as joints and fissures, many scholars adopt prefabricated fissures to simulate the weak planes in the rock mass and then use the SHPB to carry out an impact dynamic test on the rock with prefabricated fissures [32]. But in fact, the proportion of weak planes to rock is much larger than the actual proportion of weak planes in nature. Therefore, the experimental data obtained from indoor experiments are conservative and the economy is not good. It is close to the engineering practice that the rock is in different degrees of damage state by cyclic loading and unloading, and most of the rock masses in the engineering are subjected to the repeated action of the load, and the extent of the internal weak plane expansion is also related to the size of the load.

In view of the dynamic mechanical properties of damaged rock mass under impact load in geotechnical engineering, this paper adopts the cyclic loading and unloading method, which makes the rock in different degrees of damage state. Then, based on the damaged rock, the SHPB is used to carry out the impact load experiment. Finally, the dynamic strength, strain rate effect, and energy evolution of rock with different damage degrees under different strain rates are analyzed. The research results can provide some references for the safety production and stability analysis of engineering sites.

\section{Experimental Equipment and Experimental Plan}

The rock selected for this experiment was sandstone, which was taken from the rock slope of Cezi Island, Zhejiang Province, China. According to the standard of the International Society for Rock Mechanics, the sandstone was cut and polished to make the size of $\Phi 50 \mathrm{~mm}^{*} h 25 \mathrm{~mm}$. The flatness error at both ends of sandstone was not more than $0.01 \mathrm{~mm}$, and the unevenness at both ends was less than $0.05 \mathrm{~mm}$. Partially processed sandstone samples are shown in Figure 1.

This experiment was mainly divided into two steps. First of all, the processed sandstone was loaded and unloaded cyclically, made it to be damaged in different degrees. Then, a nonmetal ultrasonic detector was used to analyze the

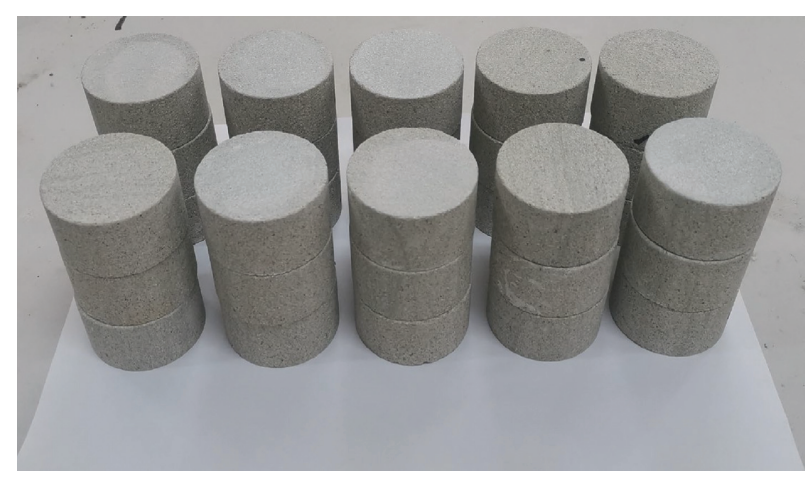

Figure 1: Partial sandstone samples.

damage degree of the rock before and after the load. Finally, SHPB was used to conduct dynamic mechanical experiments on sandstones with different degrees of damage. Therefore, it mainly involves two experimental systems, one is a static loading system, and another is a dynamic loading system.

The RMT-150C rock mechanics test system developed by the Wuhan Geotechnical Engineering Institute of the Chinese Academy of Sciences was used as the static loading system. Firstly, the uniaxial compressive strength $\sigma$ of the sandstone specimen was measured. Then, $20 \% \sigma, 40 \% \sigma$, $60 \% \sigma$, and $80 \% \sigma$ were taken as the upper limit of stress, respectively, and cyclic loading and unloading treatment was carried out on sandstone specimens. In the uniaxial compression test and cyclic loading and unloading test, the loading rate was set at $500 \mathrm{~N} / \mathrm{s}$.

The SHPB, located in Anhui University of science and technology, was used for the impact load experiment, as shown in Figure 2. The SHPB device consists of four parts. (a) The power system was composed of high-pressure nitrogen and pressure regulating device. (b) The three kinds of rods were made of Cr alloy steel with a density of $7.8 \mathrm{~g} \mathrm{~cm}^{-3}$. The elastic modulus was $210 \mathrm{GPA}$, the longitudinal wave velocity was $5190 \mathrm{~m} / \mathrm{s}$, the ultimate elastic strength is $800 \mathrm{MPa}$, and the corresponding ultimate elastic impact velocity was $40 \mathrm{~m} / \mathrm{s}$. (c) The data measurement system was composed of a strain gauge and oscilloscope. (d) Shaper, damper, and velocimeter and other auxiliary systems. The impact pressures of $0.3 \mathrm{MPa}, 0.4 \mathrm{MPa}, 0.5 \mathrm{MPa}$, and $0.6 \mathrm{MPa}$ were selected to carry out impact dynamic experiments on damaged sandstone under different upper-stress limits.

\section{Experimental Results and Analysis}

3.1. Characterization of Sandstone Damage. After the cyclic loading and unloading test, the internal defects, pores, and joints expansion of sandstone lead to the decrease of the stiffness of sandstone and eventually lead to large deformation and failure. By measuring the elastic wave velocity of rock before and after loading, the deterioration degree of sandstone under cyclic loading and unloading was quantitatively described, and the relationship between the wave velocity of rock and the upper limit of stress was obtained.

Previously, Lemaitre proposed the concept of continuous damage mechanics from the perspective of damage mechanics, based on the premise of loaded medium failure 


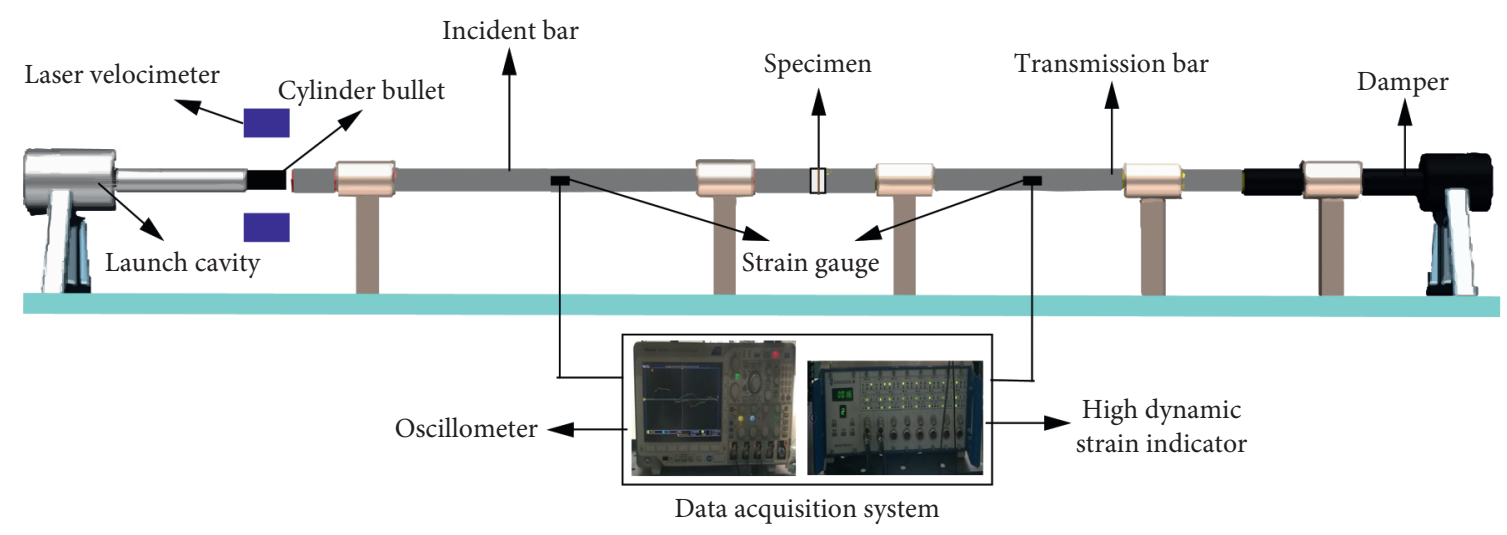

Figure 2: SHPB test device.

[33]. The damage constitutive equation based on the hypothesis of strain equivalence for a one-dimensional problem was defined as follows:

$$
\sigma_{c}=E(1-D) \mathcal{E},
$$

where $\sigma_{c}$ is the stress of nondestructive rock, MPa; $\varepsilon$ is the strain of nondestructive rock when the stress is $\sigma_{c} ; E$ is the elastic modulus of nondestructive rock, GPa; and $D$ is the damage variable, which reflects the damage degree of rock.

In the traditional damage mechanics theory, the definition method of damage factor based on this theory is the effective elastic modulus method, as shown in the following equation:

$$
D=1-\frac{E}{E_{0}},
$$

where $E$ is the elastic modulus of the damaged rock, GPa, and $E_{0}$ is the initial elastic modulus of rock, GPa. According to the inspection principle of the nonmetallic ultrasonic detector, when the ultrasonic propagation meets the internal defects of the rock, the change of the elastic modulus of the rock can be characterized by the attenuation of the ultrasonic propagation velocity. In this paper, the effective elastic modulus method in traditional mechanics is used to characterize the damage degree of loaded sandstone, and the damage factors of damaged sandstone under cyclic loading and unloading with different stress upper limits are obtained.

According to the basic theory of stress wave, the $P$-wave velocity $C_{0}$ of lossless rock and $C$ of damaged rock are shown in the following equations, respectively:

$$
\begin{gathered}
C_{0}=\sqrt{\frac{E_{0}(1-\mu)}{\rho(1-2 \mu)(1-\mu)},} \\
C=\sqrt{\frac{E(1-\mu)}{\rho(1-2 \mu)(1+\mu)}},
\end{gathered}
$$

where $\mu$ is the transverse deformation coefficient of rock; $\rho$ is the density of rock, $\mathrm{kg} / \mathrm{m}^{3} ; C_{0}$ is the $P$-wave velocity of lossless rock, $\mathrm{m} / \mathrm{s}$; and $C$ is the $P$-wave velocity of damaged rock, $\mathrm{m} / \mathrm{s}$.
Equation (5) can be obtained by combining equations (2) and (3) with (4), that is, the expression of damage factor of loaded rock:

$$
D=1-\left(\frac{C_{0}}{C}\right)^{2} .
$$

The uniaxial compressive strength of sandstone was 61.14 MPa measured by the uniaxial loading test. The sandstone specimens were in different degrees of damage state after cyclic loading with the different upper limits of stress. In this paper, the upper limit of cyclic loading and unloading stress is $12.23 \mathrm{MPa}, 24.46 \mathrm{MPa}, 36.69 \mathrm{MPa}$, and 48.92 $\mathrm{MPa}$, respectively. By measuring the wave velocity changes of sandstone specimens before and after cyclic loading and unloading and substituting equation (5) for calculation, the damage factors of sandstone with different damage degrees after cyclic loading and unloading were obtained, as shown in Table 1.

After calculation, the damage degree of sandstone under cyclic loading and unloading with different upper limits of stress was obtained. Although the stress gradient of cyclic loading and unloading was constant, the growth rate of the damage factor increased with the increase of the upper limit of stress. In practical engineering, the rock mass under the action of the load is affected by mining activities, and its internal structure is complex and changeable, resulting in different degrees of damage. The degree of rock mass disturbance is different, so the degree of damage is different. In this paper, the cyclic loading and unloading method was used to simulate the influence of different disturbance degrees on rock. When the disturbance degree is small, the structural change in the rock is small, only the primary pores are compacted and closed or part of the original defects are activated. In addition, when the upper limit of stress is 12.23 MPa, the wave velocity of a group of rocks increases. Under the action of low stress, the primary pores in the rock are compacted and closed, and the overall density increases, so the wave velocity increases. With the increase of the upper limit of stress, the structure in the loaded rock undergoes compaction and closure of the primary pores, initiation of cracks on the transition surface between the sand gravel and cemented matrix, and relative sliding between the sand gravel and the main fractures. It can be seen that the 
TABle 1: Damage factors of sandstone with different damage degrees.

\begin{tabular}{|c|c|c|c|c|}
\hline Upper limit of stress $(\mathrm{MPa})$ & $C_{0}\left(\mathrm{~m} \cdot \mathrm{s}^{-1}\right)$ & $C\left(\mathrm{~m} \cdot \mathrm{s}^{-1}\right)$ & Damage factor $D$ & Damage factor average $\bar{D}$ \\
\hline \multirow{3}{*}{12.23} & 3801.52 & 3708.11 & 0.0485 & \multirow{3}{*}{0.0321} \\
\hline & 3751.50 & 3769.70 & -0.0097 & \\
\hline & 3763.29 & 3653.29 & 0.0576 & \\
\hline \multirow{3}{*}{24.46} & 3726.92 & 3551.71 & 0.0918 & \multirow{3}{*}{0.1036} \\
\hline & 3907.95 & 3676.82 & 0.1147 & \\
\hline & 3866.39 & 3658.62 & 0.1045 & \\
\hline \multirow{3}{*}{36.69} & 3801.52 & 3133.59 & 0.3205 & \multirow{3}{*}{0.3269} \\
\hline & 3854.17 & 3231.86 & 0.2969 & \\
\hline & 3673.47 & 2930.91 & 0.3634 & \\
\hline \multirow{3}{*}{48.92} & 3775.51 & 2356.69 & 0.6103 & \multirow{3}{*}{0.6357} \\
\hline & 3814.84 & 2402.60 & 0.6033 & \\
\hline & 3726.92 & 3551.71 & 0.6918 & \\
\hline
\end{tabular}

deterioration degree of rock internal structure is gradually deepening, and the scale is also gradually increasing. Therefore, with the increase of the upper limit of stress, the growth rate of the damage factor increases gradually.

\subsection{Dynamic Compressive Strength of Damaged Sandstone.} After cyclic loading and unloading with different stress upper limits, the sandstone specimens are in different degrees of damage due to the changes in internal structure. Different types of cracks in damaged sandstone under impact load have different effects on the propagation of stress waves. Therefore, compared with nondestructive sandstone, the strength, and energy evolution of damaged sandstone under impact load are affected to varying degrees. In this paper, the impact dynamic test of sandstone with different damage degrees was carried out by using different impact pressure. Three sandstone specimens were used under each condition, and then the average value was taken. The test results are shown in Table 2.

It can be seen from the experimental results in Table 2 that the dynamic compressive strength of damaged sandstone presents an obvious strain rate effect, that is, the dynamic compressive strength of damaged sandstone increases with the increase of actuation pressure under the same upper limit cyclic load. When the actuation pressure is constant, the dynamic compressive strength of damaged sandstone decreases with the increase of damage degree.

The strength of rock under impact load usually shows an obvious strain rate effect. Some scholars believe that this is due to the change from a one-dimensional stress state to a one-dimensional strain state of rock under impact load. In addition, the generation and expansion of microcracks are also considered as an important basis. In the elastic deformation stage of rock under cyclic loading and unloading, cracks and defects that affect the propagation of stress waves begin to appear in the specimen. Under the impact load of a high strain rate, the microcracks in rock accumulate rapidly in a very short duration, which leads to the dynamic compressive strength of rock increasing with the increase of strain rate. Compared with undamaged sandstone, weak planes such as joints and fissures weaken the propagation of stress waves. In addition, the fracture of rock under impact load is caused by the expansion of some cracks. Under the impact load, the cracks in the damaged sandstone can break the rock quickly and need less energy, and the greater the damage degree is, the lower the energy is needed. Therefore, the strength of damaged sandstone under impact load is lower than that of undamaged sandstone. In order to facilitate the analysis, the strength changes of sandstone with different damage degrees under the impact load of different air pressure are fitted, as shown in Figures 3-6, respectively.

According to the test results in Figures 3-6, the dynamic compressive strength of damaged sandstone increases with the increase of actuation pressure, and at the same strain rate, the dynamic compressive strength decreases with the increase of damage degree. In addition, it can also be found that the dynamic stress variation of sandstone with the same damage degree under different actuation pressure is consistent.

The increase of impact pressure will inevitably increase the strain rate of the loaded rock. With the increase of the strain rate, the strain of the damaged sandstone will increase, which also indicates that the phenomenon of brittle decline and elastic increase appears in the sandstone. According to the dynamic mechanical properties of rock, there is a significant strain rate effect in the elastic brittle medium; that is, the mechanical characteristics of the medium under impact load are more sensitive to the strain rate or impact pressure. Therefore, the dynamic compressive strength of elastic brittle material under impact load is greater than that under uniaxial load. As a kind of brittle material, the expansion of internal fractures of sandstone is affected by the geological structure and human mining activities. In the process of underground engineering blasting, the larger the initial damage degree of rock mass is, the smaller the detonation wave generated by explosion load is.

On the basis of the test results in Table 1, the sandstone specimen has experienced the primary pore being closed by compression, the generation and expansion of new fractures, and the cross-coupling of the old and new fractures during the six cycles of loading and unloading. The damage factors of sandstone with different stress upper limit increase in $S$ shape, increase first, then decrease, and finally become stable. Under the same strain rate, the dynamic compressive strength of damaged sandstone decreases with the increase 
TABle 2: Dynamic mechanical parameters of sandstone with different damage degrees under impact load.

\begin{tabular}{|c|c|c|c|c|}
\hline Actuation pressure (MPa) & Upper limit of stress (MPa) & $\sigma_{c d}(\mathrm{MPa})$ & $\varepsilon$ & $\dot{\varepsilon}\left(\mathrm{s}^{-1}\right)$ \\
\hline \multirow{5}{*}{0.3} & 0 & 60.31 & 0.0400 & 32.14 \\
\hline & 12.23 & 48.35 & 0.1025 & 42.36 \\
\hline & 24.46 & 47.21 & 0.1234 & 45.05 \\
\hline & 36.69 & 45.06 & 0.1482 & 54.64 \\
\hline & 48.92 & 40.41 & 0.1710 & 63.52 \\
\hline \multirow{5}{*}{0.4} & 0 & 62.49 & 0.0587 & 34.32 \\
\hline & 12.23 & 51.43 & 0.1112 & 43.84 \\
\hline & 24.46 & 49.01 & 0.1302 & 48.15 \\
\hline & 36.69 & 47.59 & 0.1571 & 55.32 \\
\hline & 48.92 & 41.78 & 0.1763 & 67.62 \\
\hline \multirow{5}{*}{0.5} & 0 & 68.45 & 0.0913 & 35.32 \\
\hline & 12.23 & 56.60 & 0.1114 & 45.45 \\
\hline & 24.46 & 52.38 & 0.1330 & 49.22 \\
\hline & 36.69 & 49.64 & 0.1613 & 55.81 \\
\hline & 48.92 & 43.73 & 0.1913 & 68.22 \\
\hline \multirow{5}{*}{0.6} & 0 & 76.03 & 0.1020 & 36.58 \\
\hline & 12.23 & 59.65 & 0.1161 & 48.62 \\
\hline & 24.46 & 56.87 & 0.1346 & 50.04 \\
\hline & 36.69 & 52.86 & 0.1669 & 56.74 \\
\hline & 48.92 & 46.07 & 0.2084 & 69.25 \\
\hline
\end{tabular}

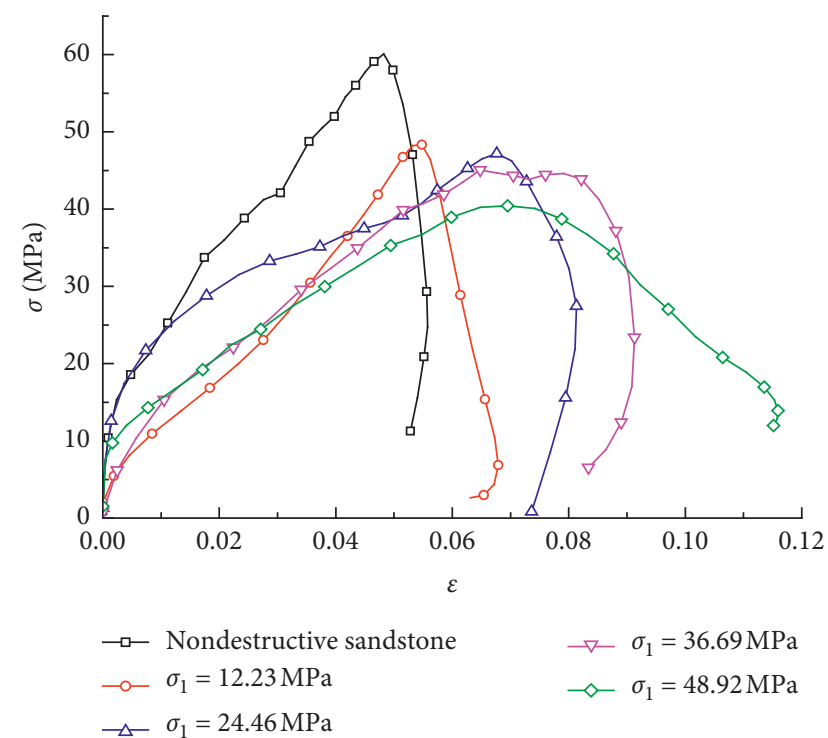

Figure 3: Dynamic stress-strain curve of damaged sandstone under impact load of $0.3 \mathrm{MPa}$ driving pressure.

of damage degree. In addition, for the damaged sandstone, the reduction rate of static compressive strength is greater than that of dynamic compressive strength. The crack produced by cyclic loading and unloading is faster than that under static load. On the other hand, the cohesive property of the medium in the damaged sandstone increases under the impact load, so the reduction rate of dynamic compressive strength is lower than that of static compressive strength.

In order to further analyze the stress evolution process of damaged sandstone under impact load, the stress-time curve and strain rate-time curve of damaged sandstone under impact load were analyzed, as shown in Figures 7 and 8,

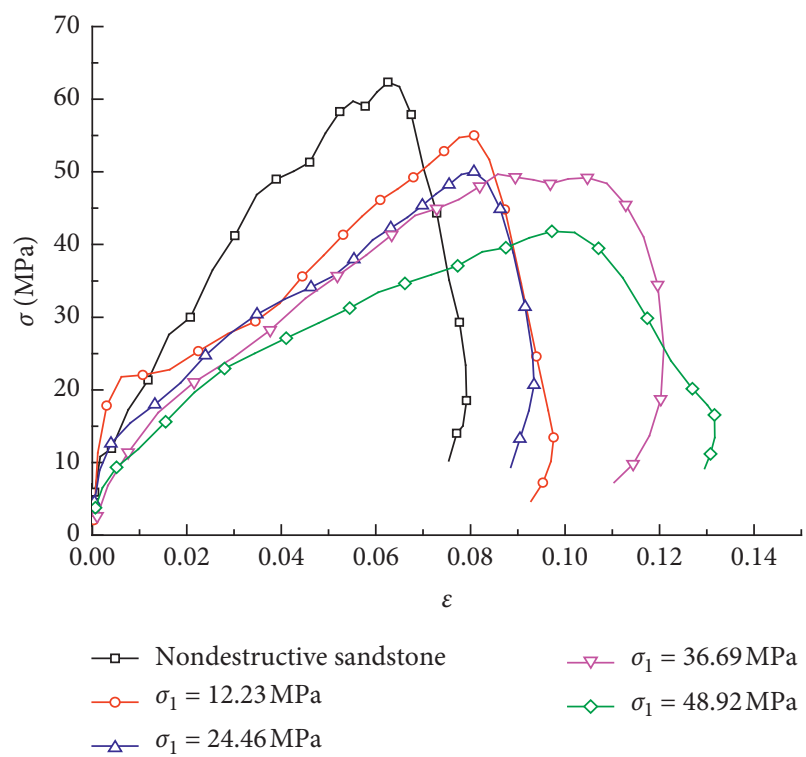

FIGURE 4: Dynamic stress-strain curve of damaged sandstone under impact load of $0.4 \mathrm{MPa}$ driving pressure.

respectively. For the convenience of analysis, this paper analyzes the stress-time history curve when the impact pressure is $0.5 \mathrm{MPa}$ only.

By analyzing the stress-time curve and strain rate-time curve of damaged sandstone under impact load, it can be seen that, with the increase of time, the variation characteristics of stress and strain rate can be divided into three stages:

(1) $0 \sim 50 \mu s$ : the changing trend of stress and strain rate is straight-line rising, and the sandstone is in the stage of stress rising. It can be seen from Figure 7 that when the upper limit of cyclic loading and unloading 


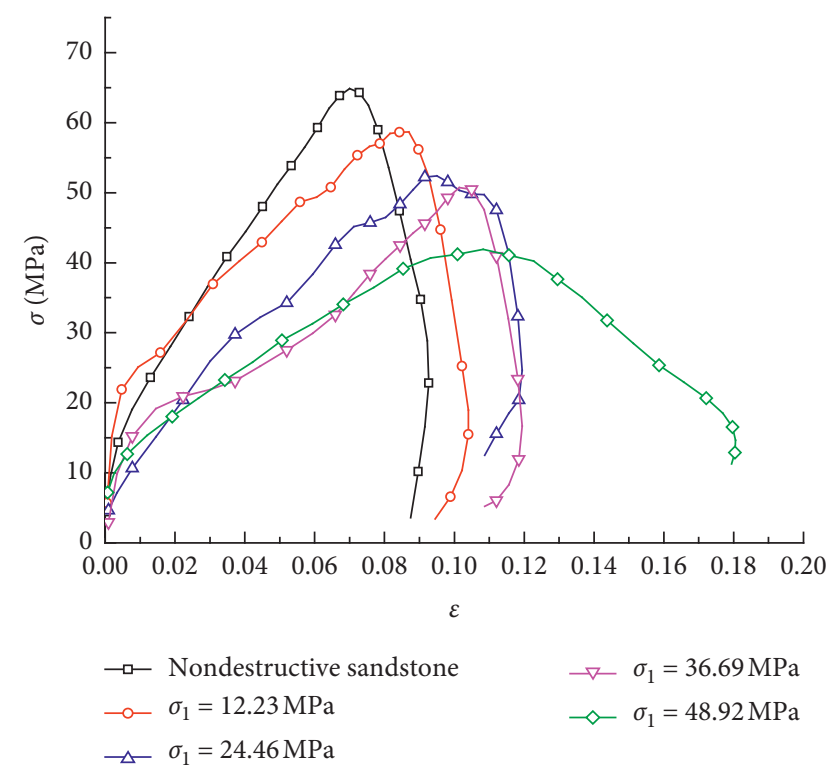

FIGURE 5: Dynamic stress-strain curve of damaged sandstone under impact load of $0.5 \mathrm{MPa}$ driving pressure.

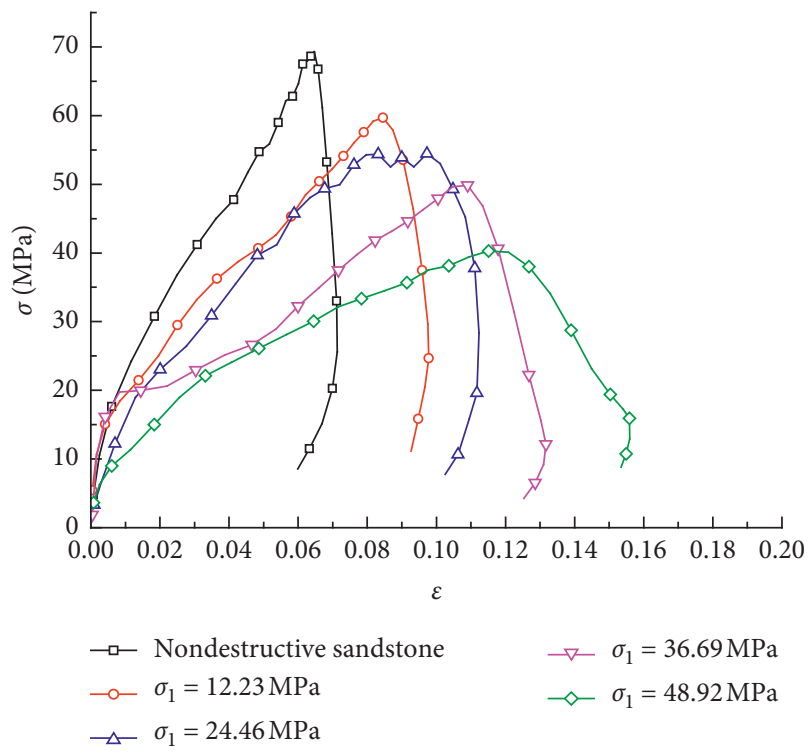

FIGURE 6: Dynamic stress-strain curve of damaged sandstone under impact load of $0.6 \mathrm{MPa}$ driving pressure.

stress is $48.92 \mathrm{MPa}$, the slope of the stress-time curve is obviously the smallest. This is because, in the cyclic loading and unloading stage, the cracks in sandstone have an impact on the propagation of stress wave under impact load. The larger the scale and the more the number of cracks, the more obvious the impact.

(2) $50 \sim 230 \mu \mathrm{s}$ : the stress increases with the increase of time, but the growth rate of stress is different when the stress increases to the peak point. The reason is that the transmission degree of the fracture to the stress wave produced by the impact load is different in the damaged sandstone with different degrees and

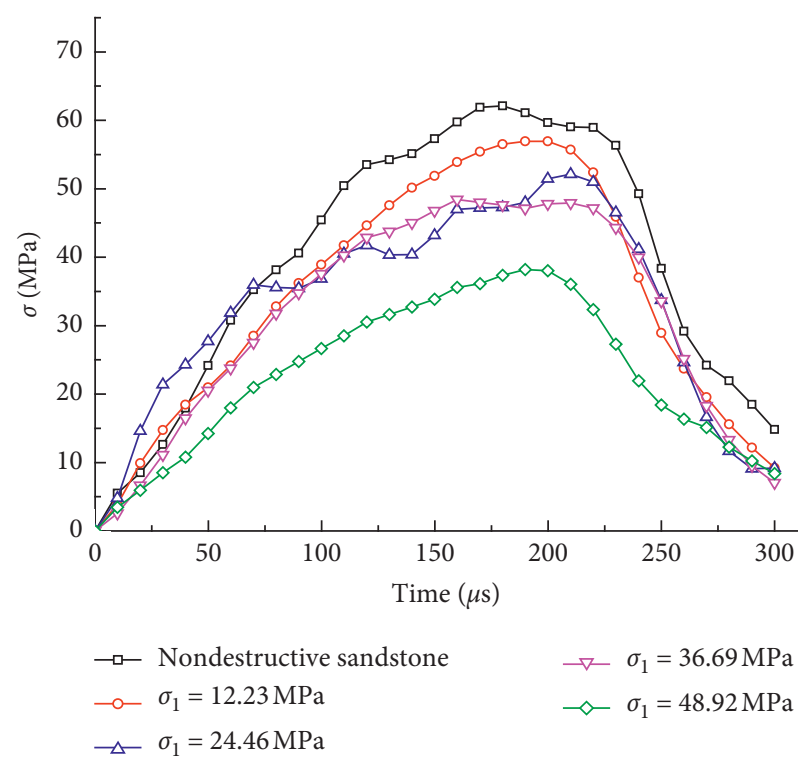

FIGURE 7: Stress-time curve of damaged sandstone under impact load.

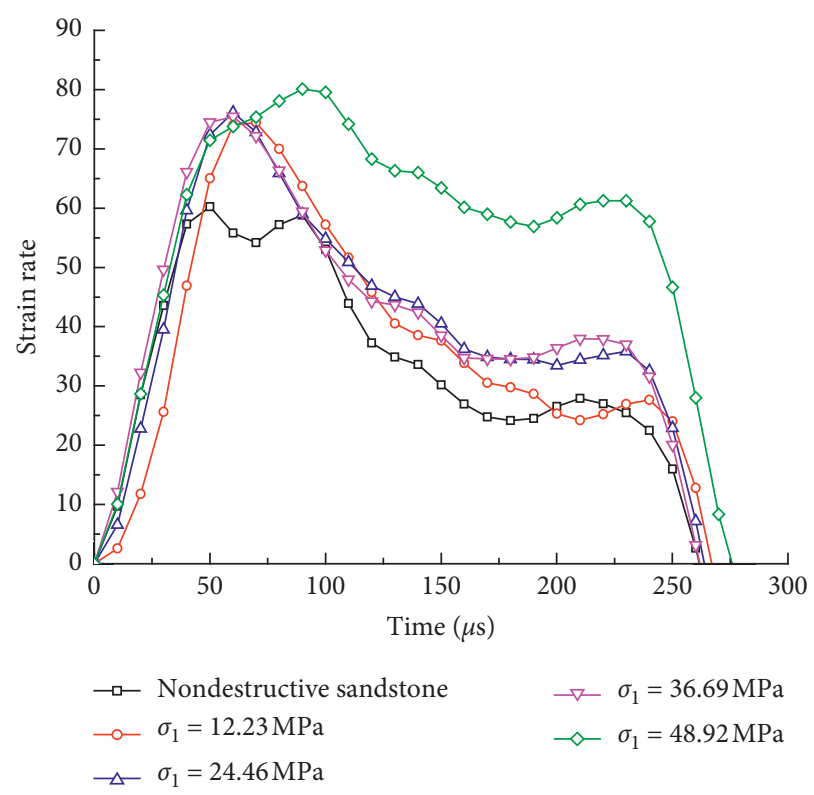

FIGURE 8: Time curve of strain rate of damaged sandstone under impact load.

scales. Although the stress changing trend of sandstone with different damage degrees is similar with time, the change degree is different. The stress growth rate of lossless sandstone is close to that of damaged sandstone whose upper-stress limit is 12.23 $\mathrm{MPa}$. The results show that the stress growth rates of sandstone specimens with an upper-stress limit of $24.46 \mathrm{MPa}$ and $36.69 \mathrm{MPa}$ are similar, and they are smaller than those of the first two kinds of sandstone specimens. When the upper-stress limit is $48.92 \mathrm{MPa}$, the stress growth rate of the damaged 
sandstone is obviously lower than that of the sandstone under the above four working conditions. The reason is that the expansion of cracks in sandstone with different damage degrees is different. The sandstone with the lower upper limit of stress after cyclic loading and unloading has less expansion degree of cracks, and the connection between cracks has not been formed. With the increase of the upper limit of stress, the expansion of new cracks leads to the connection of new cracks and old cracks from different angles, and larger cracks are produced at a faster rate. Until the upper limit of stress is 48.92 $\mathrm{MPa}$, the fractures in the sandstone expand and connect rapidly and gradually form the main fractures.

(3) $230 \sim 300 \mu s$ : the stress and strain rate of the loaded sandstone decrease continuously until the zero point. During this process, the loaded sandstone has been broken and thrown.

According to the dynamic mechanical properties of rock, the rock under impact load has an obvious strain rate effect. In order to analyze the strength change of damaged sandstone under impact load, it is necessary to analyze its strain rate effect. The strain rate effect of rock is mainly caused by the change of strain rate. The fitting curve of stress and strain rate is shown in Figure 9.

It can be seen from the fitting curve between dynamic stress and strain rate of sandstone with different damage degrees under different impact loads in Figure 9. The dynamic peak stress of sandstone is positively correlated with the strain rate. The stress-strain rate fitting curves of sandstone with different damage degrees are, respectively, shown in the following equations, which successively represent the fitting curves of damaged sandstone when the stress line is $0,12.23 \mathrm{MPa}, 24.46 \mathrm{MPa}, 36.69 \mathrm{MPa}$, and 48.92 $\mathrm{MPa}$, respectively:

$$
\begin{array}{ll}
\sigma_{c d}=44.901 e^{0.0201 \dot{\varepsilon}}, & R^{2}=0.9941, \\
\sigma_{c d}=37.878 e^{0.0179 \dot{\varepsilon}}, & R^{2}=0.9608, \\
\sigma_{c d}=37.376 e^{0.0160 \dot{\varepsilon}}, & R^{2}=0.9965, \\
\sigma_{c d}=37.709 e^{0.0130 \dot{\varepsilon}}, & R^{2}=0.9770, \\
\sigma_{c d}=34.515 e^{0.0111 \dot{\varepsilon}}, & R^{2}=0.9980,
\end{array}
$$

where $\sigma_{c d}$ is the dynamic strength of damaged sandstone under impact load, $\mathrm{MPa} ; \dot{\varepsilon}$ is the dynamic strain rate; and $R^{2}$ is the correlation coefficient of the fitting curve.

From the above fitting curves, it can be seen that the dynamic peak stress of damaged sandstone under impact load is power-related to the strain rate. The dynamic compressive strength of damaged sandstone increases with the increase of strain rate. In addition, the damage degree of sandstone also affects the strain rate of rock under impact load. It can be seen from Figure 5 that the slope of the fitting curve decreases with the increase of damage degree, that is,

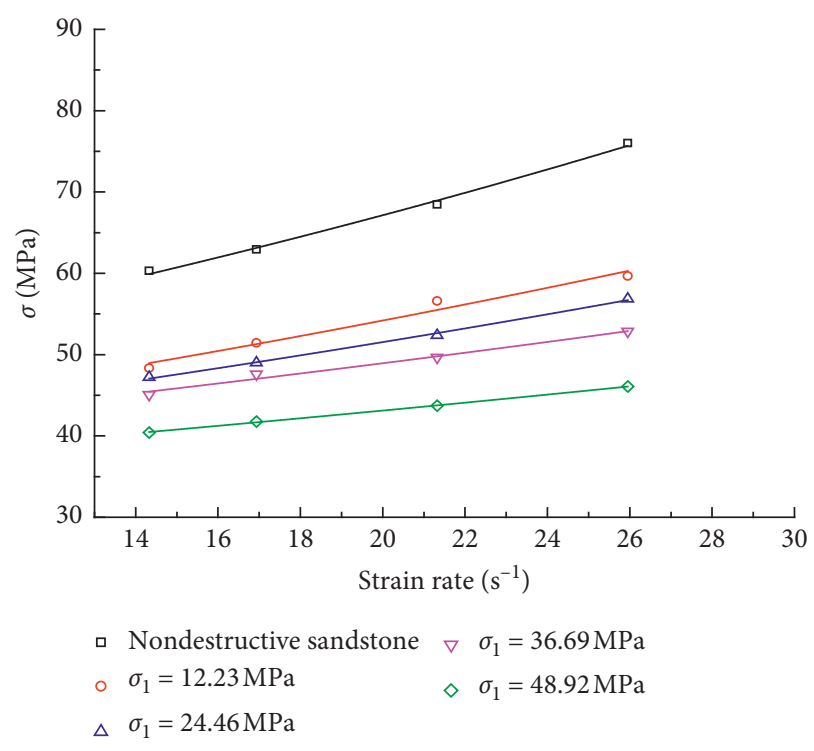

FIGURE 9: Stress-strain rate fitting curve.

the sensitivity of damaged sandstone to strain rate decreases with the increase of damage degree.

3.3. Energy Dissipation Characteristics of Damaged Sandstone under Impact Load. According to the dynamic mechanical properties of rock, the process of rock breaking under load is accompanied by the evolution of energy. There are cracks of different scales and densities in the sandstone with different degrees of damage. Under the impact load, the cracks are activated, and the rock fracture expands along the existing cracks and new cracks. In addition, the difference of fracture scale of sandstone with different damage degrees leads to different reflection and transmission of stress waves when passing through the specimen. Therefore, the energy evolution of sandstone with different damage degrees under impact load is different; that is, the reflection energy, transmission energy, and dissipation energy of sandstone with different damage degrees under the same impact load are different. The analysis of the energy evolution of sandstone with different damage degrees under impact load is also the verification of its strength change.

A simple three-wave method is used to analyze the incident energy, reflected energy, and transmitted energy of damaged sandstone under impact load, as shown in the following equations, respectively:

$$
\begin{aligned}
& W_{i}(t)=E_{0} C_{0} A_{0} \int_{0}^{t} \varepsilon_{i}^{2}(t) \mathrm{d} t, \\
& W_{r}(t)=E_{0} C_{0} A_{0} \int_{0}^{t} \varepsilon_{r}^{2}(t) \mathrm{d} t, \\
& W_{t}(t)=E_{0} C_{0} A_{0} \int_{0}^{t} \varepsilon_{t}^{2}(t) \mathrm{d} t,
\end{aligned}
$$

where $W_{i}(t), W_{r}(t)$, and $W_{t}(t)$ represent incident energy, reflection energy, and transmission energy, respectively, $J$; $E_{0}$ is the elastic modulus of sandstone specimen, GPA; $C_{0}$ is 
TABLE 3: Energy parameters.

\begin{tabular}{|c|c|c|c|c|c|}
\hline Upper limit of stress $(\mathrm{MPa})$ & $\dot{\varepsilon}\left(\mathrm{s}^{-1}\right)$ & $W_{i}(t)(\mathrm{J})$ & $W_{r}(t)(J)$ & $W_{t}(t)(\mathrm{J})$ & $W_{s}(t)(\mathrm{J})$ \\
\hline \multirow{4}{*}{12.23} & 12.74 & 10.20 & 3.89 & 4.23 & 2.08 \\
\hline & 15.77 & 30.53 & 9.32 & 17.08 & 4.13 \\
\hline & 20.16 & 52.86 & 16.86 & 24.22 & 11.78 \\
\hline & 24.88 & 79.08 & 42.22 & 16.51 & 20.35 \\
\hline \multirow{4}{*}{24.46} & 12.84 & 12.06 & 4.38 & 4.43 & 3.25 \\
\hline & 15.56 & 32.65 & 10.14 & 16.23 & 6.28 \\
\hline & 20.14 & 47.76 & 18.9 & 15.7 & 13.16 \\
\hline & 23.78 & 77.71 & 43.46 & 11.84 & 22.41 \\
\hline \multirow{4}{*}{36.69} & 12.98 & 11.02 & 5.3 & 1.06 & 4.66 \\
\hline & 15.44 & 33.88 & 12.83 & 11.61 & 9.44 \\
\hline & 19.95 & 51.84 & 22.77 & 10.55 & 18.52 \\
\hline & 24.19 & 77.14 & 48.54 & 1.54 & 27.06 \\
\hline \multirow{4}{*}{48.92} & 13.03 & 13.69 & 6.84 & 1.12 & 5.73 \\
\hline & 17.60 & 31.59 & 14.58 & 3.82 & 13.19 \\
\hline & 21.15 & 56.39 & 29.22 & 2.96 & 24.21 \\
\hline & 24.88 & 82.45 & 50.51 & 1.40 & 30.54 \\
\hline
\end{tabular}

the longitudinal wave velocity of the bar, $\mathrm{m} / \mathrm{s} ; A_{0}$ is the crosssectional area of the bar, $m^{2}$; and $\varepsilon_{i}(t), \varepsilon_{r}(t)$, and $\varepsilon_{t}(t)$ are the strain of incident wave, reflected wave, and transmitted wave propagating independently in the bar.

It is assumed that there is no energy consumption between the contact surface of sandstone and compression bar during loading, and the energy absorbed by specimen failure under impact load is shown as follows:

$$
W_{s}(t)=W_{i}(t)-W_{r}(t)-W_{t}(t) .
$$

Among all kinds of energy in sandstone specimen under impact load, $W_{s}(t)$ is the energy reflecting the fragmentation of sandstone.

According to equations (11)-(14), the incident energy, reflected energy, transmitted energy, and dissipated energy of sandstone with different damage degrees under different impact loads can be obtained, and the arrangement is shown in Table 3.

Sandstone specimens with the same damage degree can be seen from the test results in Table 3. The incident energy, reflection energy, transmission energy, and dissipation energy increase with the increase of strain rate. In addition, there is no correlation between the incident energy and the damage degree of sandstone. The magnitude of incident energy is mainly determined by the strain rate; that is, in the impact loading test, the greater the actuation pressure is, the greater the incident energy is. Compared with the incident energy, the reflected energy, transmitted energy, and dissipated energy of damaged sandstone under impact load show obvious classification with the change of damage degree. The transmission energy decreases with the increase of sandstone damage degree, while the reflection energy and dissipation energy increase with the increase of sandstone damage degree. When the upper limit of cyclic loading and unloading stress is higher, the size and scale of fracture in damaged sandstone are larger. When the stress wave passes through a large fracture, the proportion of reflected stress wave is larger, while the proportion of transmitted stress wave is smaller. Therefore, it is precise because of the difference of the size and size of cracks in the damaged sandstone after cyclic loading and unloading with different stress upper limits that the energy evolution is different with the increase of damage degree.

In the impact experiment of damaged sandstone, the reflection and transmission ratio of stress waves are different due to the different sizes and number of cracks in sandstone. According to the basic theory of rock dynamic mechanics, the magnitude of reflected energy is related to strain. On the basis of the experimental data in Table 3, with the increase of damage degree, the strain in the damaged sandstone increases significantly, which makes the reflection energy increase. In addition, the transmission energy is related to the average stress in the damaged sandstone under impact load. Therefore, with the increase of damage degree, the reflection energy of damaged sandstone increases, the transmission energy decreases, and the dissipation energy increases.

\section{Conclusions}

In this paper, the impact dynamic tests of sandstone with different damage degrees were carried out under different actuation pressure, and the dynamic strength changes and energy dissipation characteristics of sandstone with different damage degrees are analyzed. The main conclusions are as follows:

(1) The damage factor increases with the increase of the upper limit of stress.

(2) The dynamic strength and peak strain of damaged sandstone under impact load show obvious strain rate effect, both of which increase with the increase of impact pressure.

(3) The strength and peak strain of sandstone under impact load decrease with the increase of damage degree. In addition, the sensitivity of damaged sandstone to strain rate decreases with the increase of damage degree. 
(4) With the increase of damage degree of sandstone, the reflection energy and dissipation energy of sandstone increase, while the transmission energy decreases.

\section{Data Availability}

The data used to support the findings of this study are available from the corresponding author upon request.

\section{Conflicts of Interest}

The authors declare no conflicts of interest regarding the publication of this paper.

\section{Acknowledgments}

This work was funded by the National Natural Science Foundation of China (no. 52074009), patent transformation cultivation project of Anhui University of Science and Technology (no. ZL201908), and Natural Science Foundation of Anhui (no. 2008085ME163).

\section{References}

[1] F.-Q. Gong, Y. Luo, X.-B. Li, X.-F. Si, and M. Tao, "Experimental simulation investigation on rockburst induced by spalling failure in deep circular tunnels," Tunnelling and Underground Space Technology, vol. 81, pp. 413-427, 2018.

[2] X. S. Liu, J. G. Ning, Y. L. Tan, and Q. H. Gu, "Damage constitutive model based on energy dissipation for intact rock subjected to cyclic loading," International Journal of Rock Mechanics and Mining Sciences, vol. 85, pp. 27-32, 2016.

[3] Q. Zheng, H. Hu, A. Yuan et al., "Impact dynamic properties and energy evolution of damaged sandstone based on cyclic loading threshold," Shock and Vibration, vol. 2020, Article ID 6615602, 12 pages, 2020.

[4] B. L. Sainsbury and D. P. Sainsbury, "Practical use of the ubiquitous-joint constitutive model for the simulation of anisotropic rock masses," Rock Mechanics and Rock Engineering, vol. 50, no. 6, pp. 1507-1528, 2017.

[5] X. Zhang, Y. Jiang, Y. Cai, and S. Sugimoto, "Anti-plane seismic performance of a shallow-buried tunnel with imperfect interface in anisotropic half-space," Tunnelling and Underground Space Technology, vol. 112, p. 15, Article ID 103906, 2021.

[6] X. Wang, N. Wu, H. Li, and Y. Yan, "Influence of joint angle on the instability failure characteristics and AE evolution law of underground caverns," European Journal of Environmental and Civil Engineering, vol. 39, no. 5, pp. 3437-3447, 2020.

[7] X. Zhang, Y. Jiang, G. Wang, Y. Cai, and T. Iura, "Threedimensional seismic performance of mountain tunnel with imperfect interface considering $\mathrm{P}$ wave," Tunnelling and Underground Space Technology, vol. 39, Article ID 103720, 2021.

[8] B. Jiang, L. Wang, Y. Lu, S. Gu, and X. Sun, "Failure mechanism analysis and support design for deep composite soft rock roadway: a case study of the Yangcheng coal mine in China," Shock and Vibration, vol. 2015, Article ID 452479, 14 pages, 2015.

[9] W. Zeng, S.-Q. Yang, and W.-L. Tian, "Experimental and numerical investigation of brittle sandstone specimens containing different shapes of holes under uniaxial compression," Engineering Fracture Mechanics, vol. 200, pp. 430-450, 2018.
[10] R.-h. Cao, P. Cao, H. Lin, C.-z. Pu, and K. Ou, "Mechanical behavior of brittle rock-like specimens with pre-existing fissures under uniaxial loading: experimental studies and particle mechanics approach," Rock Mechanics and Rock Engineering, vol. 49, no. 3, pp. 763-783, 2016.

[11] Q. Ma, Y. Tan, X.-s. Liu, Z.-h. Zhao, and D.-y. Fan, "Mechanical and energy characteristics of coal-rock composite sample with different height ratios: a numerical study based on particle flow code," Environmental Earth Sciences, vol. 80, no. 8, p. 14, 2021.

[12] J. Xu, G. Dai, W. Gong, Q. Zhang, A. Haque, and R. P. Gamage, "A review of research on the shaft resistance of rock-socketed piles," Acta Geotechnica, vol. 16, no. 3, pp. 653-677, 2021.

[13] X. S. Liu, J. G. Ning, Y. L. Tan, and Q. H. Gu, "Coordinated supporting method of gob-side entry retaining in coal mines and a case study with hard roof," Geomechanics and Engineering, vol. 15, no. 6, pp. 1173-1182, 2018.

[14] S.-q. He, L.-z. Jin, S.-n. Ou, and X.-h. Ming, "Soft coal solidgas coupling similar material for coal and gas outburst simulation tests," Journal of Geophysics and Engineering, vol. 15, no. 5, pp. 2033-2046, 2018.

[15] Q.-q Zheng, Y. Xu, H. Hu, J.-w Qian, Q. Zong, and P. Xie, "Fracture and tomography of velocity structures of sandstone under uniaxial loads," Chinese Journal of Geotechnical Engineering, vol. 43, no. 6, pp. 1069-1077, 2021.

[16] Y. Xu, Q. Zheng, X. Gao, R. Yang, X. Ni, and Q. Wang, "Quantitative damage and fracture mode of sandstone under uniaxial load based on acoustic emission," Advances in Civil Engineering, vol. 2020, Article ID 6685795, 9 pages, 2020.

[17] Q. Zheng, Y. Xu, H. Hu, J. Qian, Y. Ma, and X. Gao, "Quantitative damage, fracture mechanism and velocity structure tomography of sandstone under uniaxial load based on acoustic emission monitoring technology," Construction and Building Materials, vol. 272, Article ID 121911, 13 pages, 2021.

[18] Q. Zheng, Y. Cheng, Q. Zong, Y. Xu, F. Li, and P. Chen, "Failure mechanism of different types of shotcrete based on modified Weibull distribution model," Construction and Building Materials, vol. 224, pp. 306-316, 2019.

[19] J. Wang, J. G. Ning, P. Q. Qiu, S. Yang, and H. F. Shang, "Microseismic monitoring and its precursory parameter of hard roof collapse in longwall faces: a case study," Geomechanics and Engineering, vol. 17, no. 4, pp. 375-383, 2019.

[20] L. Li, I. Larsen, and R. M. Holt, "Laboratory observation and micromechanics-based modelling of sandstone on different scales," Rock Mechanics and Rock Engineering, vol. 48, no. 4, pp. 1407-1422, 2015.

[21] Z. Zhou, X. Cai, X. Li, W. Cao, and X. Du, "Dynamic response and energy evolution of sandstone under coupled static-dynamic compression: insights from experimental study into deep rock engineering applications," Rock $\mathrm{Me}$ chanics and Rock Engineering, vol. 53, no. 3, pp. 1305-1331, 2020.

[22] S. Song, X. Liu, Y. Tan, D. Fan, Q. Ma, and H. Wang, "Study on failure modes and energy evolution of coal-rock combination under cyclic loading," Shock and Vibration, vol. 2020, Article ID 5731721, 16 pages, 2020.

[23] B. P. Simser, "Rockburst management in Canadian hard rock mines," Journal of Rock Mechanics and Geotechnical Engineering, vol. 11, no. 5, pp. 1036-1043, 2019.

[24] K. Xia, W. Yao, and B. Wu, "Dynamic rock tensile strengths of Laurentian granite: experimental observation and 
micromechanical model," Journal of Rock Mechanics and Geotechnical Engineering, vol. 9, no. 1, pp. 116-124, 2017.

[25] H. Hu, Q. Zheng, X. Gao, B. Cheng, Q. Wang, and X. Ni, "Fracture characteristics and geometric fractal of damaged sandstone under impact load," Shock and Vibration, vol. 2020, Article ID 6617197, 10 pages, 2020.

[26] O. Y. Vorobiev and M. B. Rubin, "Modeling the dynamic response of rock masses with multiple compliant fluid saturated joint sets-part I: mesoscale simulations," International Journal of Impact Engineering, vol. 151, p. 15, Article ID 103747, 2021.

[27] K. Xia and W. Yao, "Dynamic rock tests using split Hopkinson (Kolsky) bar system-a review," Journal of Rock Mechanics and Geotechnical Engineering, vol. 7, no. 1, pp. 27-59, 2015.

[28] S. Mishra, T. Chakraborty, and R. K. Seshagiri, "Dynamic response of two extrusive igneous rocks using split Hopkinson pressure bar test," Journal of Materials in Civil Engineering, vol. 33, no. 6, p. 14, Article ID 04021133, 2021.

[29] Z. Yan, F. Dai, Y. Liu, A. Li, and H. Du, "Numerical assessment of the rate-dependent cracking behaviours of single-flawed rocks in split Hopkinson pressure bar tests," Engineering Fracture Mechanics, vol. 247, p. 16, Article ID 107656, 2021.

[30] A. Kumar, "The effect of stress rate and temperature on the strength of basalt and granite," Geophysics, vol. 33, no. 3, pp. 501-510, 1968.

[31] Y. Wang, X. Li, and B. Zheng, "Stress-strain behavior of soilrock mixture at medium strain rates-response to seismic dynamic loading," Soil Dynamics and Earthquake Engineering, vol. 93, pp. 7-17, 2017.

[32] J. Huang, X. Liu, J. Zhao, E. Wang, and S. Wang, "Propagation of stress waves through fully saturated rock joint under undrained conditions and dynamic response characteristics of filling liquid," Rock Mechanics and Rock Engineering, vol. 53, no. 8, pp. 3637-3655, 2020.

[33] J. Lemaitre and J. Dufailly, "Damage measurements," Engineering Fracture Mechanics, vol. 28, no. 5, pp. 643-661, 1987. 